\title{
NUMERICAL MODELLING OF WAVE INTERACTION WITH DYNAMICALLY STABLE STRUCTURES
}

\author{
Marcel R.A. van Gent ${ }^{1}$
}

\begin{abstract}
Wave interaction with dynamically stable structures is simulated by means of a numerical model based on finite-amplitude shallow-water wave equations. The model can simulate wave motion on and inside permeable structures. For dynamically stable structures, including berm breakwaters, reef-type structures and gravel beaches, a procedure is developed to simulate the natural response to wave attack. This procedure is extended, for instance by implementing effects of grading and effects of seawalls, to increase the applicability for practical applications.
\end{abstract}

\section{INTRODUCTION}

Coastal structures that consist of rubble mound material and are designed to undergo reshaping under severe storm conditions, while their seaward slope is stable under normal wave conditions, are called here dynamically stable structures. These structures such as berm breakwaters and reef-type structures can be economically attractive since their natural response to hydrodynamic loads causes that smaller rock material can be used than with conventional statically stable coastal structures. On the other hand, the dynamic behaviour needs to be predicted to assess the performance of such structures. For berm breakwaters this dynamic behaviour of the seaward slope is very much depending on the hydrodynamic loads and vice versa. This interactive character of the hydrodynamics and the reshaping process are studied here by means of a numerical wave load-response model.

A verified wave model has been combined with a procedure to simulate the response of dynamic structures (Van Gent, 1995-c). Procedures for initiation of movement of individual stones and for the reshaping of the structure as a result of moving stones determine the response of the structure. Both the wave motion and the response of the structure are simulated in the time-domain which means that the response of a structure immediately effects the computed wave motion.

1) Delft Hydraulics, P.O.Box 177, 2600 MH Delft, The Netherlands. 


\section{MODELLING OF WAVE ATTACK}

A numerical model for simulating wave motion on and inside permeable structures is described in Van Gent $(1994,1995-b, c)$. The wave dynamics of normally incident wave attack on various types of structures are approximated by the non-linear shallow-water wave equations with steep wave fronts represented by bores. The model is based on concepts by Hibberd and Peregrine (1979) who developed a numerical model with an explicit dissipative finite-difference scheme (Lax-Wendroff) for impermeable slopes without friction. Using this concept, many practical applications have been obtained, see for instance Kobayashi et al. (1987) for wave reflection and run-up on impermeable rough slopes. For the permeable part of a structure the same types of equations can be applied although the friction coefficients for porous media needed to be assessed, see Van Gent (1995-a,c). The coupling of the external flow to the internal flow is determinative for the accuracy of such a wave model, see Van Gent (1994, 1995-c).

The model is able to deal with waves either regular or irregular which attack various types of structures with arbitrary seaward slopes, smooth or rough, permeable or impermeable, overtopped or not. Although the model uses a onedimensional description of the flow it can, however, give a useful impression of the flow field in two dimensions, see Figure 1 and Figure 2.

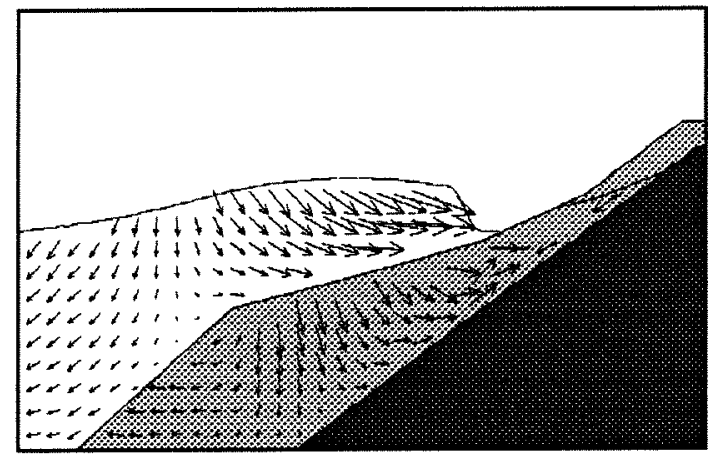

Fig.1 Computed flow-field for a berm breakwater with an impermeable core.

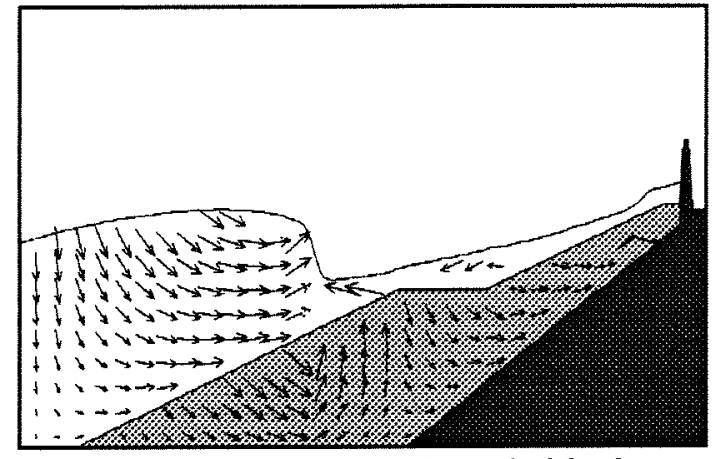

Fig.2 Computed flow-field for a permeable breakwater with crown-wall.

\section{MODELLING OF RESHAPING OF STRUCTURES}

\subsection{APPROACH FOR SIMULATING PROFILE DEVELOPMENT}

The stability of the stones is strongly dependent on the hydrodynamic properties. Several expressions for this stability have been developed. Although these design recommendations are rather accurate for many applications, more generally applicable results can be obtained by simulating the wave motion first and then using flow properties like the velocities and accelerations to predict forces on stones. This can be done numerically. 
In the approach towards a numerical wave load-response model several model formulations are required. Firstly, the hydrodynamic flow, both outside and inside the structure, need to be known and modelled numerically. The mentioned onedimensional model can be used as a first approximation. Secondly, information concerning the magnitude of forces on stones is necessary. Thirdly, relations between the forces on stones and the hydrodynamic behaviour are necessary. As mentioned before, the hydrodynamics can be represented by local velocities and local accelerations. As a first approximation, a Morison-type of expression (Morison et al., 1950) can be used, see for instance Kobayashi and Otta (1987) or Tørum (1992). Fourthly, information concerning failure mechanisms and forces causing damage is needed. Often failure mechanisms referred to as rolling, sliding or lifting are distinguished. These mechanisms or other failure mechanisms need to be modelled. Finally, the new positions of unstable stones need to be known if the complete reshaping process is to be simulated. For most breakwaters no severe damage is allowed, so for those cases it is not of primary interest to study the new positions of the stones. However, for berm breakwaters and gravel beaches these new positions are of primary concern. Only the main aspects of the wave loadresponse model are mentioned in the following sections. A more detailed description, including the calibration and validation of the model, can be found in Van Gent (1995-c).

\subsection{MODELLING OF FORCES ON STONES}

The hydrodynamic loadings on a single stone can be modelled using a number of forces. For the relation between the hydrodynamics and the forces, local velocities and local accelerations are required. The numerical model provides these local properties although averaged over the depth. Differences between these properties at the position of the particles and the depth-averaged velocities naturally cause inaccuracies.

Three forces as a result of the hydrodynamic loadings have been distinguished; the drag force acting parallel to the slope in the direction of the velocity, the inertia force acting parallel to the slope and the lift force acting perpendicular to the slope:

$$
\begin{aligned}
& F_{D}=\frac{1}{2} \rho c_{D} k_{2} D^{2} u|u| \\
& F_{I}=\rho c_{M} k_{1} D^{3} \frac{D u}{D t} \\
& F_{L}=\frac{1}{2} \rho c_{L} k_{2} D^{2} u^{2}
\end{aligned}
$$

where the acceleration $D u / D t$ is approximated with $\partial u / \partial t ; c_{D}, c_{M}, c_{L}$ are the drag coefficient, the inertia coefficient and the lift coefficient respectively; $k_{1}$ and $k_{2}$ are the volume shape factor and the area shape factor respectively. With the area shape 
factor $k_{2}$ the actual projected area in the flow direction can be incorporated. Since a cover particle is partially sheltered by other particles, the actual projected area is smaller than for a single particle in a flow. The sheltering effect has not been incorporated separately and therefore affects the values of the coefficients which are derived through calibration. In all computations $k_{1}=0.66$ and $k_{2}=0.9$ were used. For the stone diameter the equivalent sphere diameter $D_{E Q}$ is taken as the characteristic stone size $\left(D_{E Q} \approx 1.24 \cdot D_{n 50}\right)$.

The submerged weight is taken as the counter-acting force. The submerged weight acts vertically and can be written as ( $\rho_{s}$ is the density of the stone material):

$$
W_{s}=\left(\rho_{s}-\rho\right) g k_{1} D^{3}
$$

Several concepts can be used for initiation of movement. Stability criteria for the phenomena referred to as lifting and sliding can respectively be expressed by:

$$
\begin{aligned}
& F_{L} \leq W_{s} \cos \phi \\
& \left|F_{D}+F_{I}-W_{s} \sin \phi\right| \leq \tan \mu\left(W_{s} \cos \phi-F_{L}\right)
\end{aligned}
$$

where $\mu$ denotes the angle of internal friction and $\phi$ the local slope angle. The phenomenon rolling is assumed to occur if both stability conditions are not satisfied.

\subsection{MODELLING OF STONE DISPLACEMENTS}

If at a certain position a stone in the slope is unstable, the direction in which the stone will move has to be determined. It is assumed that the drag and inertia forces and the weight of the stone determine in which direction an unstable particle will move after one of the stability criteria is (Eq.5 and/or Eq.6) not satisfied:

$$
\begin{aligned}
& \left(F_{D}+F_{I}-W_{s} \sin \phi\right)>0 \Rightarrow \text { UPWARD } \\
& \left(F_{D}+F_{I}-W_{s} \sin \phi\right)<0 \Rightarrow \text { DOWNWARD }
\end{aligned}
$$

In Van Gent (1995-c) erroneously an additional force $\left(F_{P}\right)$ was taken into account in equations 7 and 8 . Although this force did not have any effect on the computations, this force has been omitted in the new computations.

After determining the direction in which an unstable particle may move, the local hydrodynamic properties at a position one space-increment $(\Delta x)$ away from the original position, are regarded. It is verified whether the particle would be stable or unstable in that neighbouring position. If the particle is stable at that position, the particle will stay at its original position. If the particle is also unstable at the 
neighbouring position the particle is moved to this position. This is done without any time-delay which means that the particle is moved over a space-increment $\Delta x$ within a period of $\Delta t$. The choice of $\Delta t$ depends on the space-increment $\Delta x$ and the wave celerity which means that the velocity of the stones is in fact related to the (average) wave celerity.

This response model for cross-structure transport is interactive with the hydraulic model. At each time-step $(\Delta t)$ the hydraulic properties are determined at all positions. At each position and each time-step it is verified whether the particles are stable at their present position or not and whether they need to be displaced. The profile changes due to the movement of the particles while the new profile is immediately incorporated in the hydraulic model.

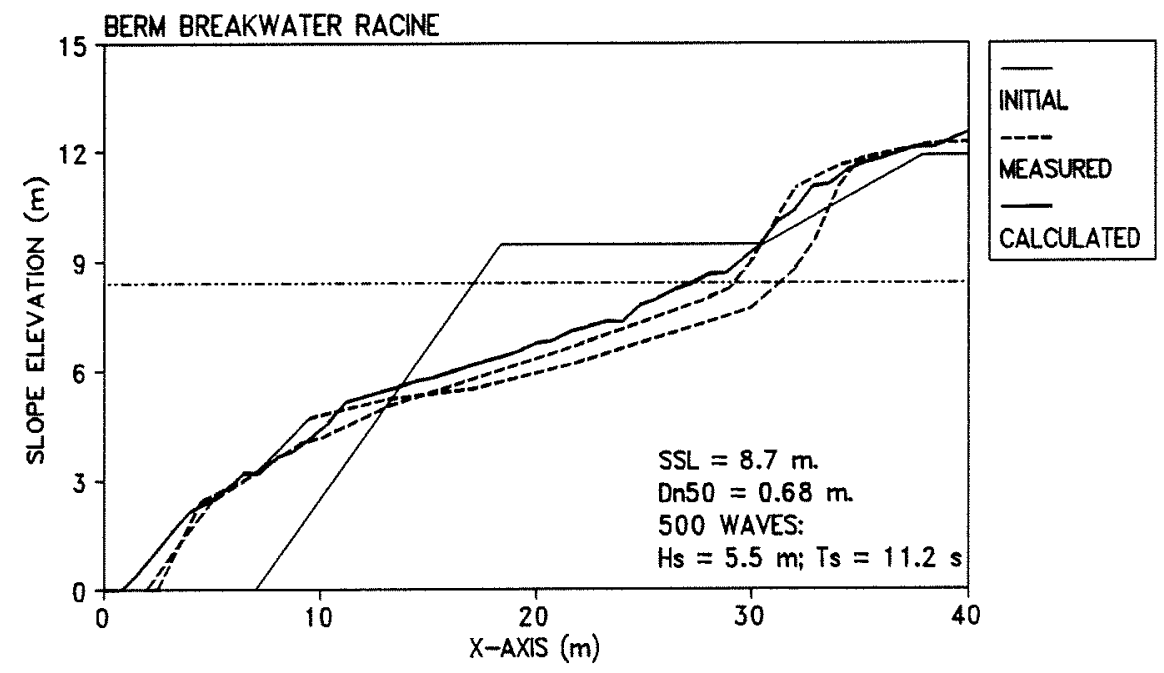

Fig.3 Comparison between measured and computed reshaped profiles of a prototype berm breakwater.

A few examples of performed computations are shown here. Figure 3 shows a comparison between measured (Montgomery et al, 1988) and computed reshaped slopes of a (prototype) berm breakwater. Computations for reef-type structures were performed after verification of wave transmission coefficients. Tests by Ahrens (1987) were used for comparisons of the dynamical response of reef-type structures, see Figure 4.

\section{MODELLING OF EFFECTS OF GRADING}

Besides a quantitative validation of the model also trends that have been observed in physical model tests have been studied. This concerns the effects of wave height, wave period, stone size and initial slopes. The wave load-response model has been extended to also include the effects of variations in stone size along the slope and stone size segregation. 

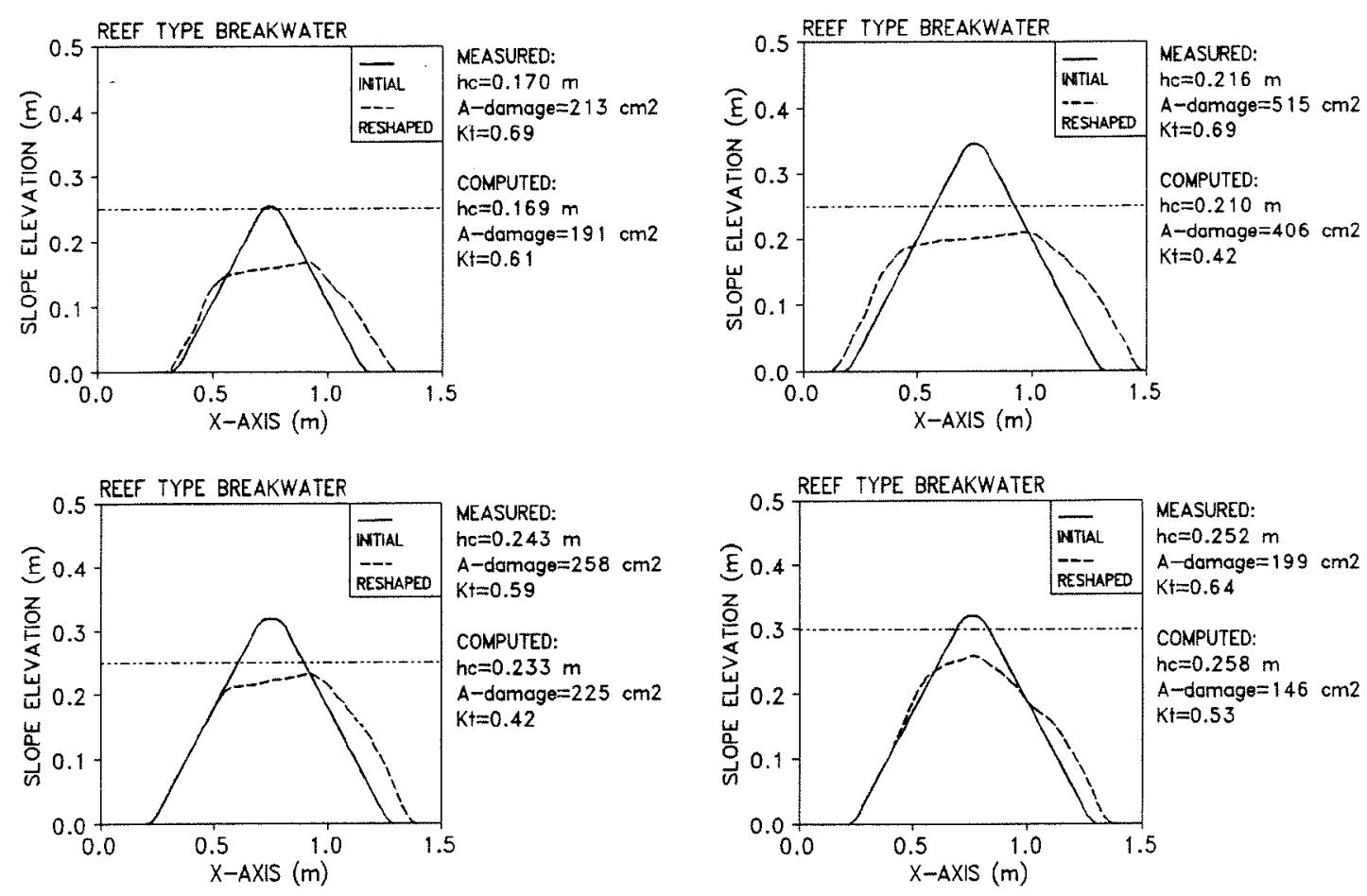

Fig.4 Computations with reef-type structures, initial profiles and reshaped profiles (stable).

To include these effects of the stone size distribution, the initial slope is made out of stones with at each position (each $\Delta x$ ) a stone with a different size. This is done randomly but such that the slope has a prescribed grading curve. At each position the forces are determined on the stone with its particular size. If a stone is unstable and moved in the upward or downward direction, the stone (size) in the second layer is treated as the exposed stone. At the position where the stone is moved to, this stone becomes the exposed stone while the originally exposed stone becomes the stone in the second layer. In this treatment the thickness of the layers is not equal to one stone diameter. Despite this simplification the effects of material with a range of sizes can be studied numerically.

The effect of grading on the reshaping process is not only determined by the size distribution of the stones. A wider stone size grading also has a lower permeability than a narrow grading. Grading might also affect the friction but since this phenomenon is not sufficiently quantified, the friction has not been varied in these computations $(f=0.10)$.

The reshaping of gravel beaches with three different gradings are computed; $D_{n 85} / D_{n 15}=1.0,1.33$ and 2.0. For the grading curve a linear distribution is taken. Because the original wave load-response model was calibrated on a physical model test with material with a grading of $D_{n 85} / D_{n 15}=1.5$, while in the numerical model a grading of $D_{n 85} / D_{n 15}=1.0$ was used, the coefficients $c_{L}, c_{M}$ and $c_{D}$ should in fact 
have been determined again after implementing the effect of grading. Since no recalibration was done, the analysis performed here is concentrated on the effects of grading itself and not into detail on the magnitude of the erosion and accretion on the reshaped profiles.

In Figure 5 reshaped profiles of three gravel beaches are shown. First only the effect of permeability is studied. For gradings of $D_{n 85} / D_{n 15}=1.0,1.33$ and 2.0 the effect of decreasing permeability has been taken into account by simply reducing the porosity from $n=0.45$ for $D_{n 85} / D_{n 15}=1.0$ to $n=0.40$ for $D_{n 85} / D_{n 15}=1.33$ and $n=0.35$ for $D_{n 85} / D_{n 15}=2.0$. The results of this variation in permeability is shown in the upper graph of Figure 5. The second graph shows the effect of the variation in stone sizes along the slope while in the lower graph both effects are simultaneously taken into account. It is clear from the figures that reducing the permeability leads to more erosion of the slope. This seems reasonable since a increased permeability reduces the velocities along the slope. The variation in the size distribution shows a counteracting effect; a wider distribution shows a less affected profile. Apparently, after some reshaping the larger material of the distribution forms the toplayer and reduces further reshaping of the slope.

Combining the two effects shows (lower graph of Figure 5) that for the narrow grading the effect of lower permeability is slightly larger than the effect of the wider size distribution (slight increase in erosion from $D_{n 85} / D_{n 15}=1.0$ to 1.33 ). For wider gradings the effect of the wider size distribution becomes slightly more important (slight decrease in erosion from $D_{n 85} / D_{n 15}=1.33$ to 2.0 ). Similar conclusions were obtained by Kao and Hall (1990) who studied the effects of grading on a reshaping berm breakwater through physical modelling.

Figure 6 shows the computed effects of three different gradings for reshaped profiles with different material size $\left(D_{n 50}\right)$. As can be expected, larger material leads to less erosion of the initial slope; this also leads to a smaller effect of a wider gradation for larger material. Figure 7 shows the effects on reshaped profiles for different initial slopes; steeper initial slopes are much more affected than milder slopes. For slopes where considerable erosion of the initial slope occurs again the effects of a wider gradation is obvious. Apparently, the larger material of the distribution forms the exposed layer after some reshaping and reduces further reshaping of the slope. The computations also show that for wider gradings more material is moved upward; the forming of a beach crest is more substantial for wider gradings while the forming of a submerged bar is more apparent for narrow gradings.

Since the motion of material with different sizes within one slope is modelled, also the effect of stone segregation is simulated. Figure 8 shows the stone size distribution of material along the slope after reshaping. The stone size distribution is given for the exposed stones and also for the average of the ten upmost stones. In Figure 8 the stone size material is averaged over some sections of the slope in 
which the variation in size is relatively small. Two examples for different initial slopes are shown in Figure 8. The general conclusion is that in the region around and just below the still-waterline the material in the toplayer is relatively large. More downward and more upward from this region around the still-waterline, the material is somewhat smaller. The main conclusion is that after reshaping relatively large material is located in the region where the largest velocities occur, i.e. just below the still-waterline.

\section{MODELLING OF EFFECT OF TOP-LAYER WITH HIGHER WEIGHT}

In practice sometimes the largest stones from a quarry are positioned on top of the primary armour layer of a berm breakwater. This causes that initial reshaping occurs for somewhat more severe wave conditions than without this measure. By using the numerical model it is studied whether positioning the largest stones on top has effect on the final reshaped profile. The computations shown in Figure 9 indicate that these largest stones are moved either upward or downward and do not remain in the section around the waterline were the largest velocities occur. This also causes that the final reshaped profile is almost not affected.

\section{MODELLING OF EFFECTS OF A SEAWALL}

Some computations are performed with a fully reflecting impermeable boundary landward from the gravel beach. If such a seawall is positioned in the region that is affected by reshaping under conditions without a seawall it is obvious that the seawall can change the reshaping process. Since a one-dimensional model is applied here for conditions where vertical accelerations cannot always be neglected, the results are affected by extra inaccuracies and must not be taken too seriously.

Figure 10 shows results for three different initial slopes. For each initial slope the first reshaped profile shows the situation without a seawall, the second profile the effect of a seawall located on the beach and the third profile the effect of a seawall at the initial waterline. In general the trend is that the seawall causes the submerged bar to develop more seaward, as can be expected since the reflected waves cause increased velocities in the seaward direction. The computations do not show a clear local effect on the reshaped profile near the seawall; in some computations the local erosion increases slightly but in some other computations some piling-up of stones against the seawall occurs.

\section{CONCLUDING REMARKS}

The reshaping of dynamic structures such as berm breakwaters, reef-type structures and gravel beaches has been simulated numerically. This approach 

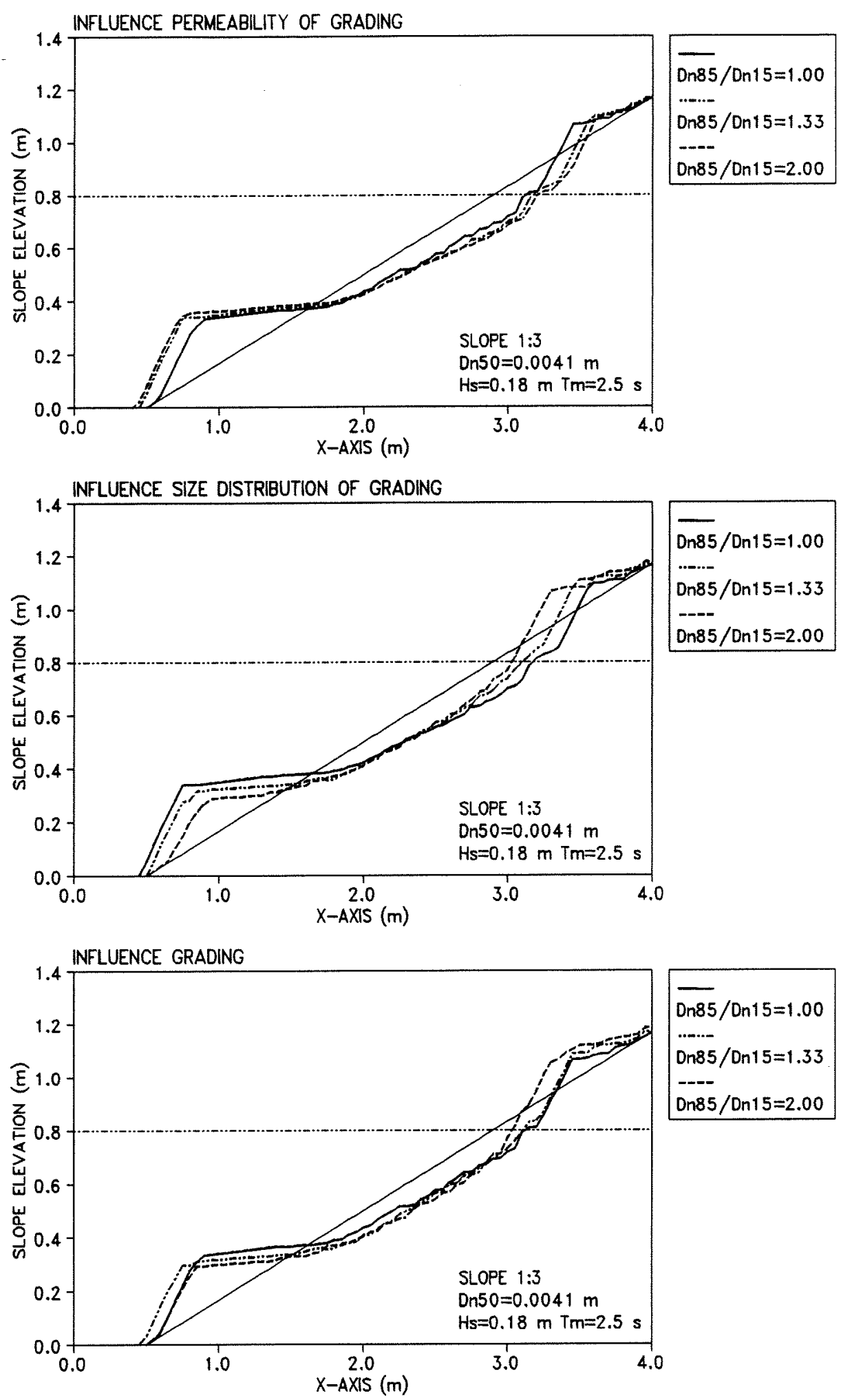

Dn85/On15=1.00

……

Dn85/Dn15=1.33

Dn85/Dn15 $=2.00$

Fig.5 Influence of permeability, size distribution and combined effects of grading on reshaped profiles. 

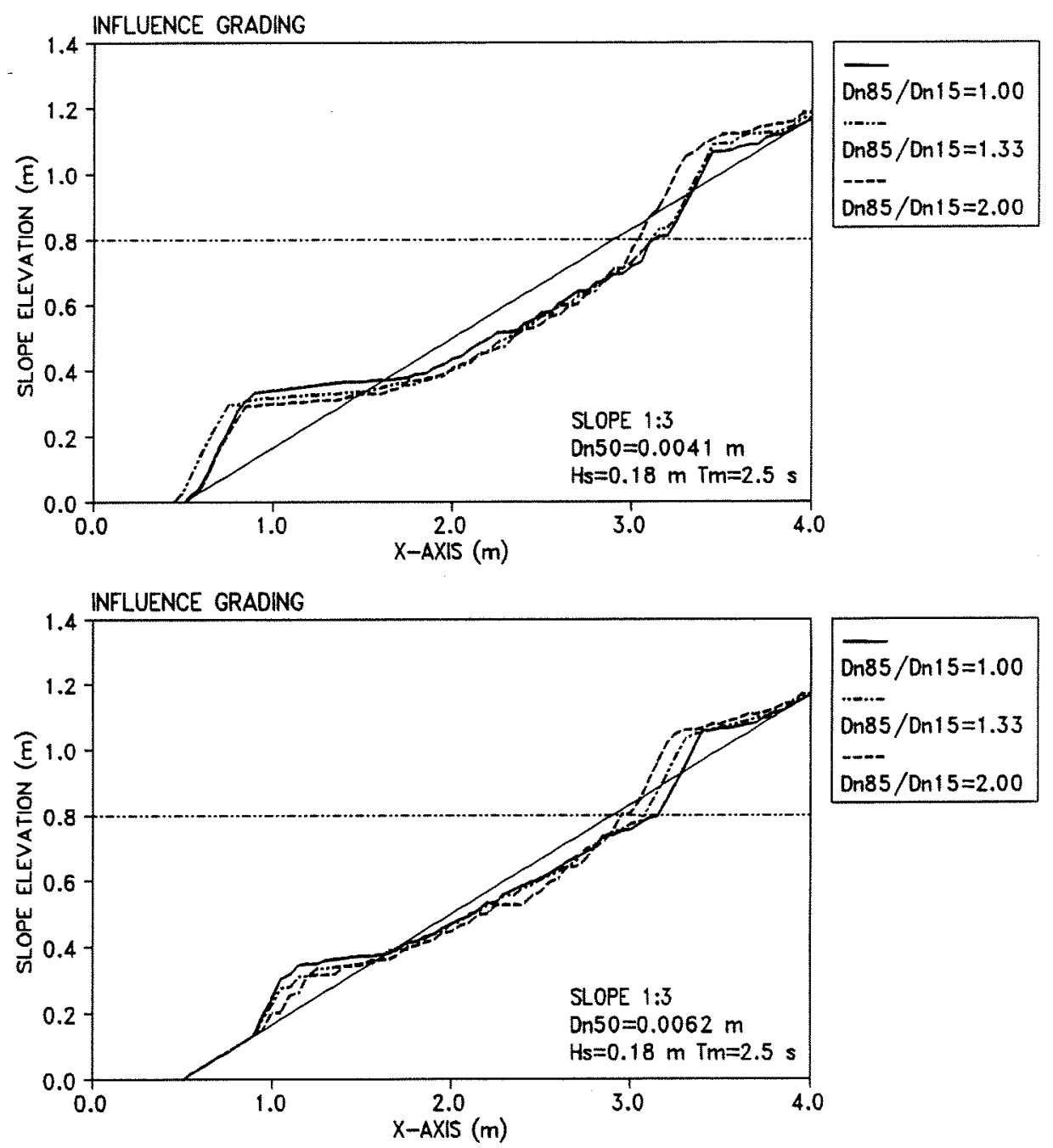

Dn85/Dn $15=1.00$

Dn85/Dn $15=1.33$

$-\cdots$

Dn85/Dn15 $=2.00$

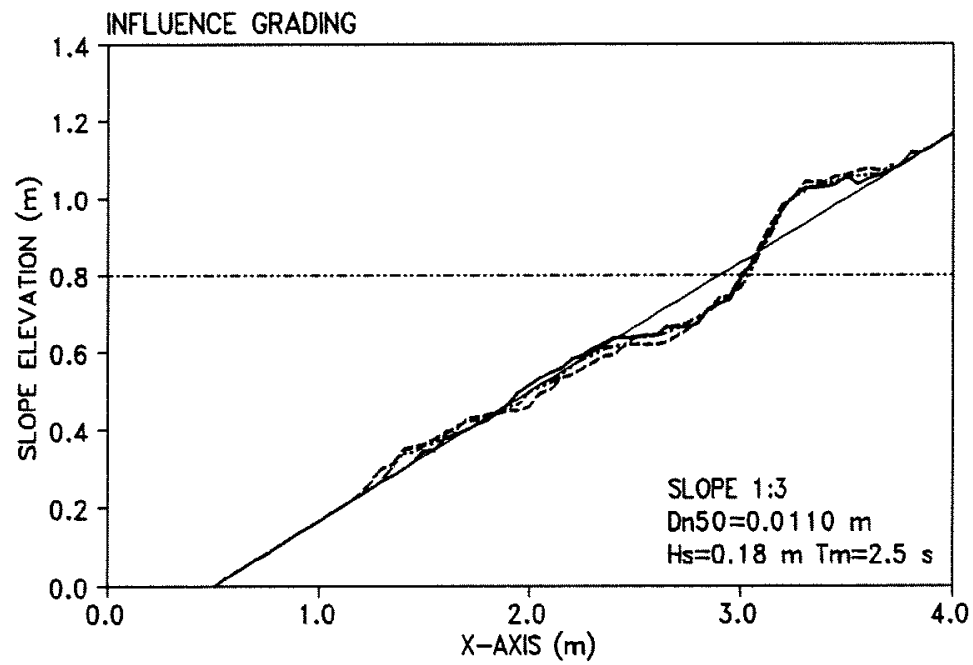

Dn85/Dn $15=1.00$

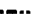

Dn85/Dn $15=1.33$

Dn85/Dn15 $=2.00$

Fig.6 Influence of grading on reshaped profiles for different stone sizes. 

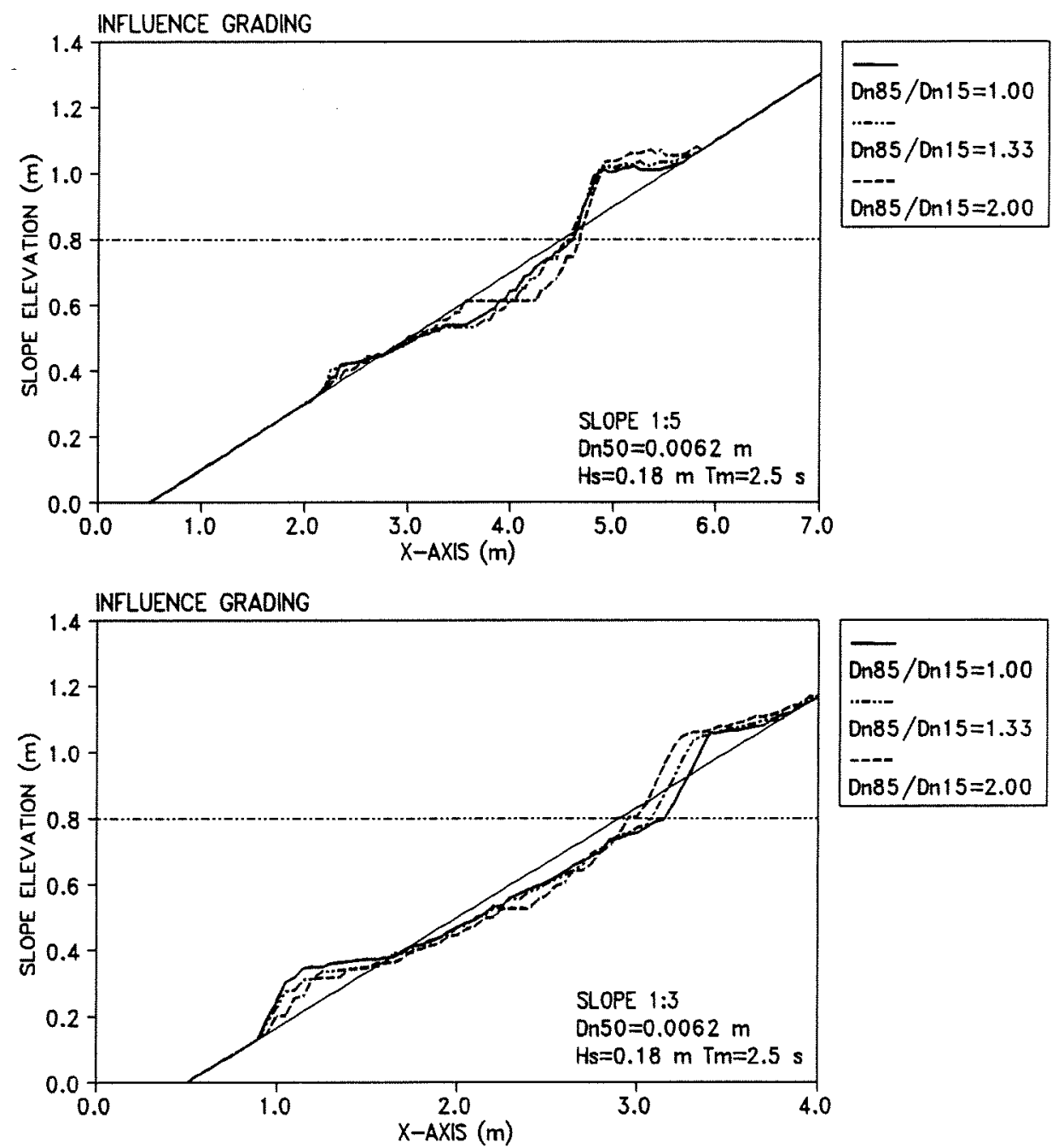

Dn85/Dn15 $=1.00$ Dn85/Dn15=1.33

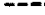

Dn85/Dn $15=2.00$

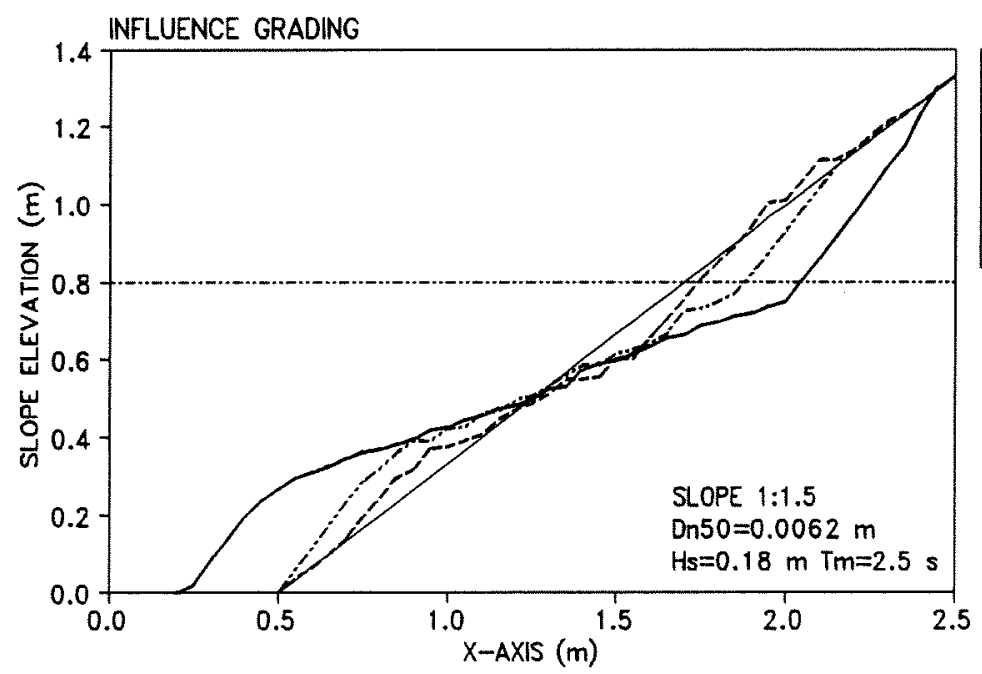

Dn85/Dn $15=1.00$ Dn85/Dn $15=1.33$

Dn85/Dn $15=2.00$

Fig.7 Influence of grading on reshaped profiles for different slope angles. 

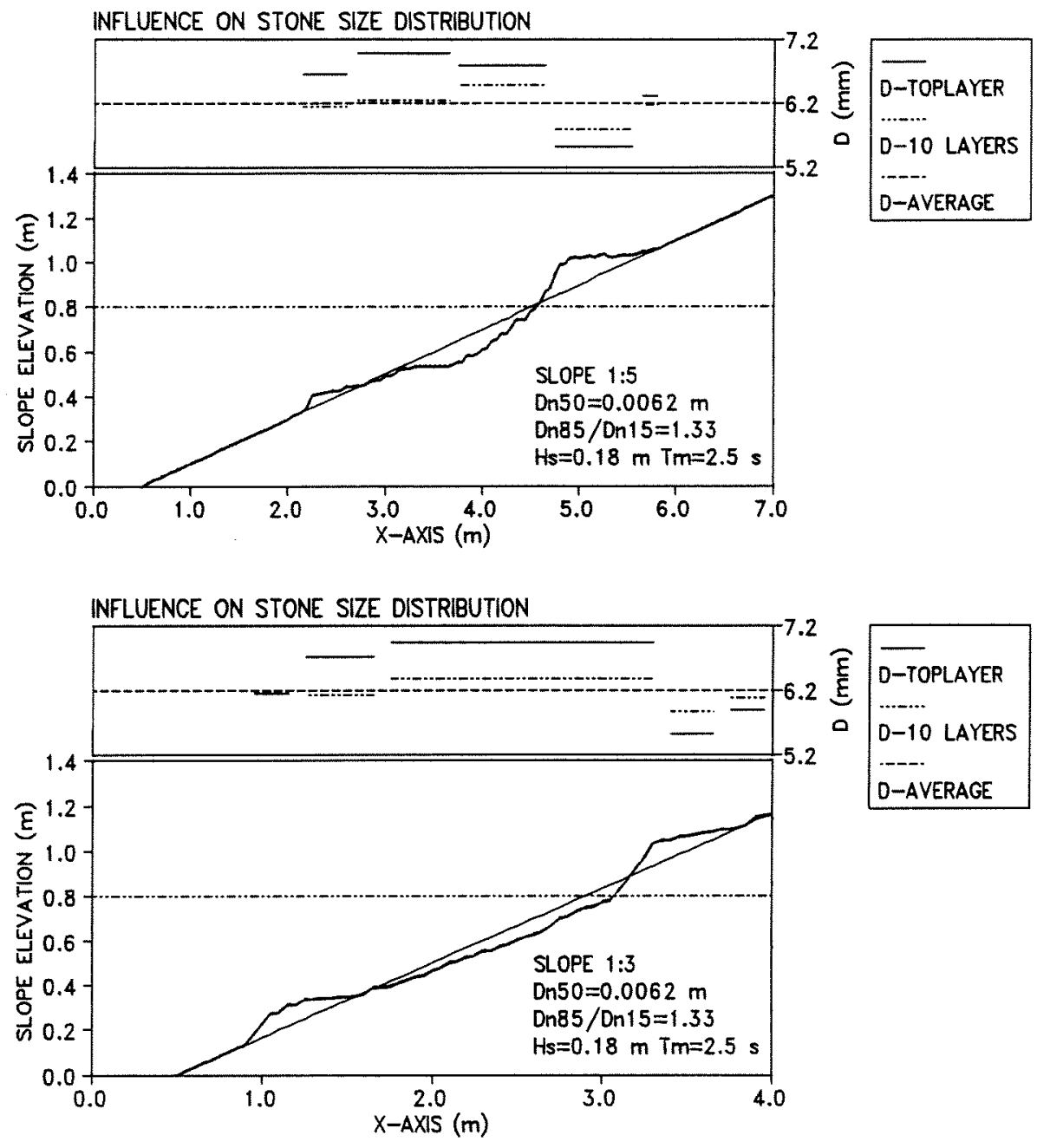

Fig.8 Stone size distribution along reshaped slopes.

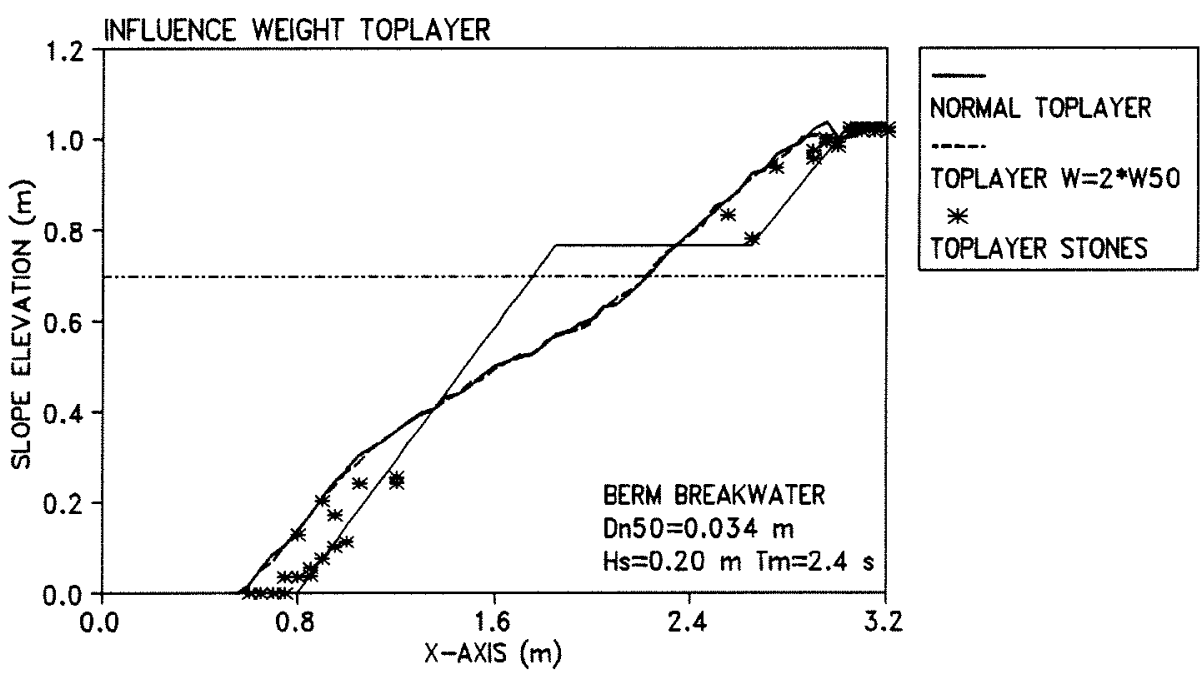

Fig.9 Influence of an increased weight of toplayer stones. 

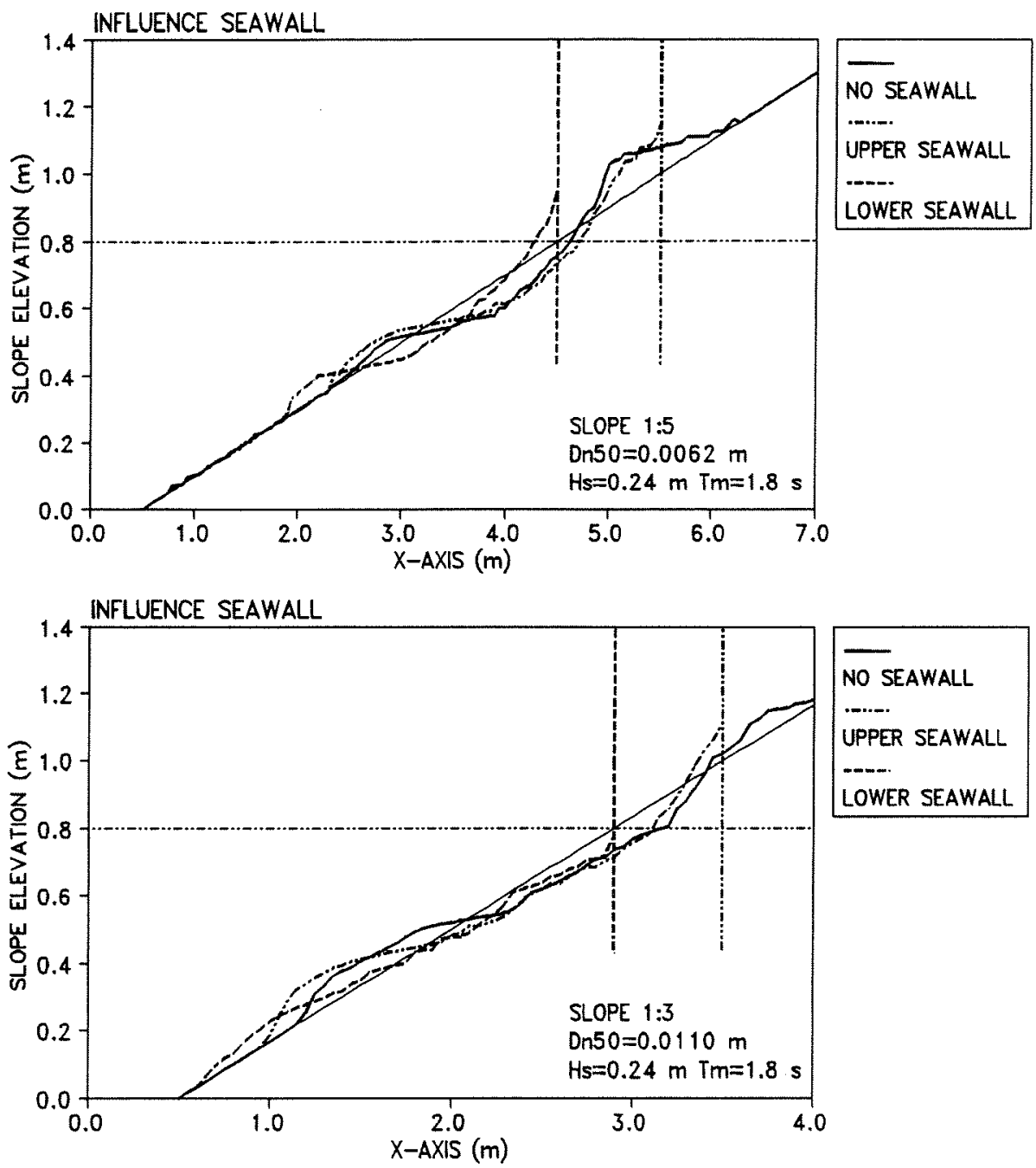

NO SEAWALL

UPPER SEAWALL

LOWER SEAWALL

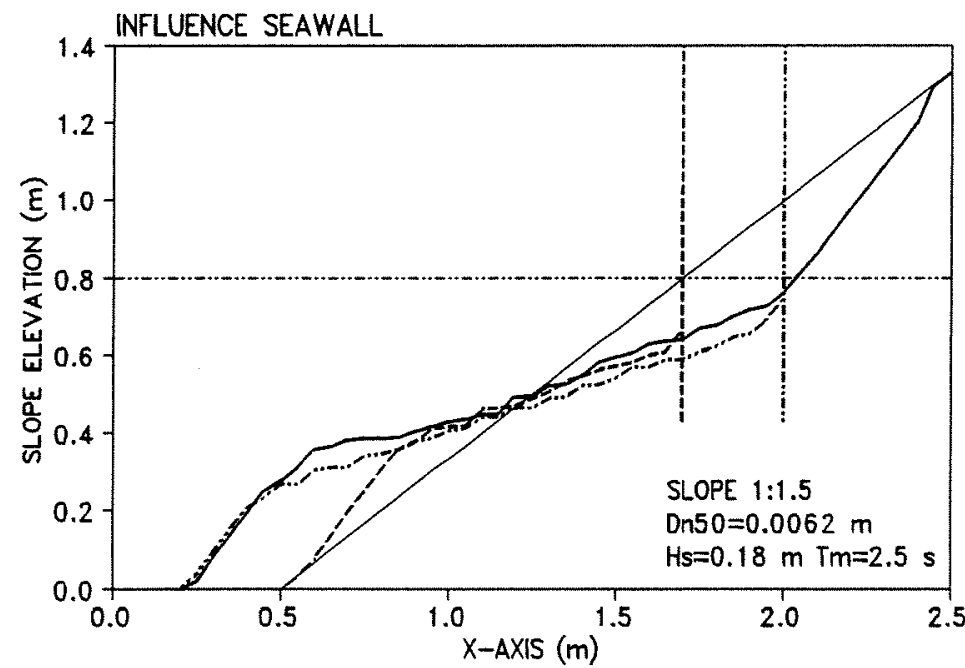

NO SEAWALL

UPPER SEAWALL

LOWER SEAWALL

Fig.10 Influence of a reflecting seawall in front of a reshaping profile. 
appeared to be successful for predicting trends in the effects of variations of the wave height, wave period, stone material and initial slope. Although not validated, also effects of grading of stone material and effects of a seawall on a beach were incorporated. Further improvements to this wave load-response model might be obtained by applying a model which provides more detailed and more accurate information on the wave motion; the two-dimensional model (2DV) described in Van Gent et al. (1994, 1995-c), which includes detailed modelling of breaking waves, can be applied but this requires considerable computational efforts.

\section{ACKNOWLEDGEMENTS}

The financial support by the Commission of the European Communities by way of the MAST-Berm Breakwater project (contract MAS2-CT94-0087) is gratefully acknowledged. Thanks are also due to Prof. K. d'Angremond from the Delft University of Technology since a large part of the research concerning this paper is performed at this University.

\section{REFERENCES}

Ahrens, J.P. (1987), Characteristics of reef breakwaters, Report CERC-87-17, Vicksburg. Hibberd, S. and D.H. Peregrine (1979), Surf and run-up on a beach: a uniform bore, J. of Fluid Mechanics, Vol.95, part 2, pp.323-345.

Gent, M.R.A. van (1994), The modelling of wave action on and in coastal structures, Coastal Engineering, Vol.22 (3-4), pp.311-339, Elsevier, Amsterdam.

Gent, M.R.A. van, P. Tönjes, H.A.H. Petit and P. van den Bosch (1994), Wave action on and in permeable structures, Proc.ICCE'94, Vol.2, pp.1739-1753, Kobe.

Gent, M.R.A. van (1995-a), Porous flow through rubble mound material, J. of Waterway, Port, Coastal and Ocean Engineering, ASCE, Vol.121, no.3, pp.176-181.

Gent, M.R.A. van (1995-b), Wave interaction with berm breakwaters, J. of Waterway, Port, Coastal and Ocean Engineering, ASCE, Vol.121, no.5, pp.229-238.

Gent, M.R.A. van (1995-c), Wave interaction with permeable coastal structures, Ph.D.thesis, Delft University of Technology, ISBN 90-407-1182-8, Delft University Press.

Kao, J.S. and K.R. Hall (1990), Trends in stability of dynamically stable breakwaters, Proc. ICCE'90, Vol.2, pp.1730-1741, Delft.

Kobayashi, N., A.K. Otta and I. Roy (1987), Wave reflection and run-up on rough slopes, J. of Waterway, Port, Coastal and Ocean Engrg, ASCE, Vol.113, no.3, pp.282-298.

Kobayashi, N. and A.K. Otta (1987), Hydraulic stability analysis of armour units, J. of Waterway, Port, Coastal and Ocean Engineering, ASCE, Vol.113, No.2, pp.171-185.

Morison, J.R., M.P. O'Brien, J.W. Johnsen and S.A. Schaff (1950), The forces exerted by surface waves on piles, Petrol. Trans. AIME, Vol.189, pp.149-154.

Montgomery, R.J., G.J. Hofmeister and W.F. Baird (1988), Implementation and performance of berm breakwater design at Racine, W, Berm breakwaters; (ed. D.H. Willis et al.), derived from Workshop Ottawa, September 1987, pp.230-249.

Tørum, A (1992), Wave induced water particle velocities and forces on an armour unit on a berm breakwater, MAST-G6S report STF60-A92104, NHL-Trondheim, Norway. 